\title{
Rate Enhancement of Diels-Alder Reactions in Aqueous
}

\section{Perfluorinated Emulsions}

\author{
Kohsuke Nishimoto, Shokaku Kim, Yoshikazu Kitano, Masahiro Tada, Kazuhiro Chiba* \\ Laboratory of Bio-organic Chemistry, Tokyo University of Agriculture and Technology, \\ 3-5-8 Saiwai-cho, Fuchu, Tokyo, 183-8509, Japan \\ chiba@cc.tuat.ac.jp
}

\section{Preparation of LiFOS}

Water was purified by a Milli-Q Water-Purification System. The perfluorinated emulsions were prepared with LiFOS and PFH in water under sonication for $1 \mathrm{hr}$ at ambient temperature. After the completion of the emulsion formation, the dispersion was partially applied for the measurement of the particle size distributions (NIKKISO Nanotrac UPA-EX150) at $25^{\circ} \mathrm{C}$.

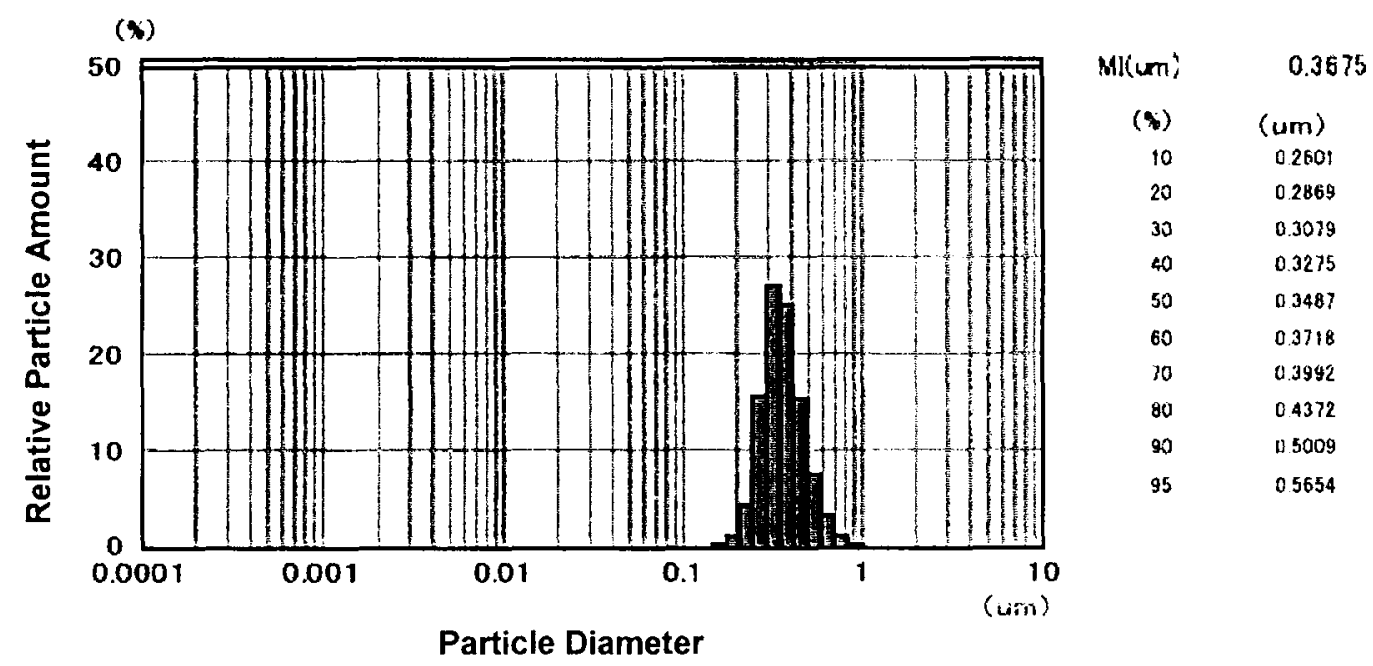


<smiles>CC1=C(C)C[C@]2(C)C(=O)c3ccccc3C(=O)C2C1</smiles>

\section{3: 2,3,4a-Trimethyl-1,4,4a,9a-tetrahydro-anthraquinone}

${ }^{1} \mathrm{H}-\mathrm{NMR}\left(\mathrm{CDCl}_{3}, 600 \mathrm{MHz}\right) \delta: 8.08-7.98(2 \mathrm{H}, \mathrm{m}), 7.76-7.70(2 \mathrm{H}, \mathrm{m}), 3.02(1 \mathrm{H}, \mathrm{dd}, J=5.5,7.7), 2.60$ $(1 \mathrm{H}, \mathrm{d}, J=17.6), 2.42(1 \mathrm{H}, \mathrm{dd}, J=7.7,17.6), 2.17(1 \mathrm{H}, \mathrm{dd}, J=5.5,17.6), 1.79(1 \mathrm{H}, \mathrm{d}, J=17.6), 1.63(3 \mathrm{H}, \mathrm{s})$, $1.61(3 \mathrm{H}, \mathrm{s})$ and $1.34(3 \mathrm{H}, \mathrm{s}) ;{ }^{13} \mathrm{C}-\mathrm{NMR}\left(\mathrm{CDCl}_{3}, 150 \mathrm{MHz}\right) 8: 200.3,198.6,134.2,134.0,133.7,133.1$, $127.3,126.6,123.6,122.7,53.7,48.5,38.7,31.2,23.8,18.9$ and 18.6<smiles>CC1=C(C)CC2C(=O)c3ccccc3C(=O)C2C1</smiles>

\section{5: 2,3-Dimethyl-1,4,4a,9a-tetrahydro-anthraquinone}

${ }^{1} \mathrm{H}-\mathrm{NMR}(\mathrm{CDCl} 3,600 \mathrm{MHz}) \delta: 8.04(2 \mathrm{H}, \mathrm{dd}, J=3.4,5.8), 7.74(2 \mathrm{H}, J=3.4,5.8), 3.39-3.33(2 \mathrm{H}, \mathrm{m})$, 2.50-2.40 (2H, m), 2.19-2.09 (2H, m), 1.64(6H, s); ${ }^{13} \mathrm{C}-\mathrm{NMR}\left(\mathrm{CDCl}_{3}, 150 \mathrm{MHz}\right) \delta: 198.4,134.2,134.1$, $126.8,123.5,47.4,30.7$ and 18.9 


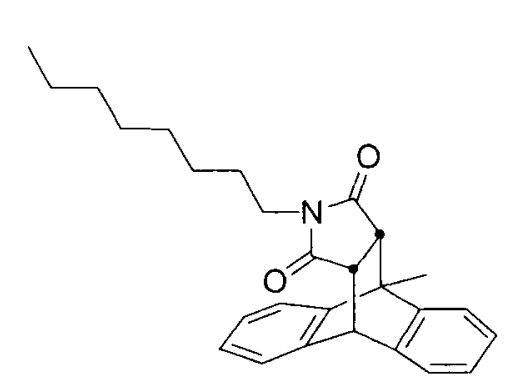

8: 3a,4,9,9a-Tetrahyd ro-4-methyl-2-octyl-4,9[1',2']benzo-1H-benz[f] isoindole-1,3-(2H)-dione

${ }^{1} \mathrm{H}-\mathrm{NMR}\left(\mathrm{CDCl}_{3}, 400 \mathrm{MHz}\right)$ 8:7.41-7.34 (2H, m), 7.30-7.24 (2H, m), 7.24-7.08 (4H, m), $4.76(1 \mathrm{H}, \mathrm{d}$, $J=3.2), 3.21(1 \mathrm{H}, \mathrm{dd}, J=3.2,8.3), 3.07(2 \mathrm{H}, \mathrm{t}, J=7.3), 2.80(1 \mathrm{H}, \mathrm{d}, J=8.3), 2.29(3 \mathrm{H}, \mathrm{s}), 1.36-1.01(8 \mathrm{H}$, $\mathrm{m}), 0.88(3 \mathrm{H}, \mathrm{t}, J=7.1)$ and $0.86-0.66(4 \mathrm{H}, \mathrm{m}) ;{ }^{13} \mathrm{C}-\mathrm{NMR}\left(\mathrm{CDCl}_{3}, 150 \mathrm{MHz}\right) \delta: 176.67,176.01,144.60$, $141.93,141.20,138.80,126.76,126.58,126.42,126.40,124.90,123.71,122.13,121.91,50.31,48.20$, $45.49,44.92,38.31,31.72,28.96,28.88,27.07,26.36,22.57,15.24$ and $14.04 ;$ MS (EI) m/z(relative intensity \%) $401\left(\mathrm{M}^{+}, 4\right), 302(23)$ and $192(100)$; IR (KBr) 3068, 3039, 3023, 2923, 2871, 2852, 1772, $1708,1465,1438,1398,1361,1338,1174,1141,1022,773,763,754$ and $709 \mathrm{~cm}^{-1}$

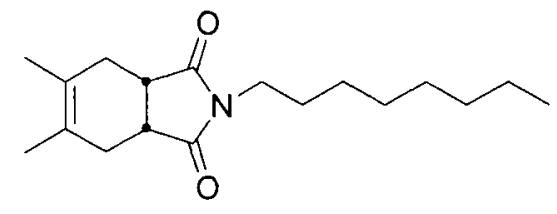

\section{9: 5,6-Dimethyl-2-octyl-3a,4,7,7a-tetrahydro-isoindole-1,3-dione}

${ }^{1} \mathrm{H}-\mathrm{NMR}\left(\mathrm{CDCl}_{3}, 400 \mathrm{MHz}\right) \delta: 3.39(2 \mathrm{H}, \mathrm{t}, J=7.1), 2.94(2 \mathrm{H}, \mathrm{t}, J=2.4), 2.38(2 \mathrm{H}, \mathrm{d}, J=14.9), 2.16(2 \mathrm{H}$, bd, $J=14.9), 1.65(6 \mathrm{H}, \mathrm{s}), 1.42(2 \mathrm{H}$, quint,$J=7.3) 1.28-1.00(10 \mathrm{H}, \mathrm{m})$ and $0.81(3 \mathrm{H}, \mathrm{t}, J=6.8) ;{ }^{13} \mathrm{C}-\mathrm{NMR}$ $\left(\mathrm{CDCl}_{3}, 150 \mathrm{MHz}\right) \delta: 180.27,126.81,39.76,38.77,31.71,30.74,29.13,29.08,27.55,26.42,22.54$, 19.15 and 13.99 ; HRMS calcd. for $\mathrm{C}_{18} \mathrm{H}_{29} \mathrm{O}_{2} \mathrm{~N} \mathrm{~m} / \mathrm{z} 291.2198$ found $\mathrm{m} / \mathrm{z} 291.2176 ; \mathrm{MS}$ (EI) $\mathrm{m} / \mathrm{z}$ (relative intensity \%) 29l( $\left.\mathrm{M}^{+}, 100\right), 276(5), 193(16), 108(58)$ and 82(2) ; IR (NaCl) 2927, 2856, 1768, 1700, $1438,1400,1371,1349$ and $1174 \mathrm{~cm}^{-1}$ 
<smiles>CCOC(=O)C12C=CC(=O)C(O)=C1CC(C)=C(C)C2</smiles>

11: Ethyl 8-hydroxy-2,3-dimethyl-7-oxo-1,4,4a,7-tetrahydronaphthalene-4a-carboxylate

${ }^{1} \mathrm{H}-\mathrm{NMR}\left(\mathrm{CDCl}_{3}, 600 \mathrm{MHz}\right)$ 8:6.95 (1H, d, $\left.J=9.9\right), 6.50-6.45(2 \mathrm{H}, \mathrm{m}), 4.21-4.03(2 \mathrm{H}, \mathrm{m}), 3.35(1 \mathrm{H}, \mathrm{d}$, $J=20.9), 2.95(1 \mathrm{H}, \mathrm{d}, J=20.9), 2.93(1 \mathrm{H}, \mathrm{d}, J=16.1), 2.07(1 \mathrm{H}, \mathrm{d}, J=16.1), 1.70(3 \mathrm{H}, \mathrm{s}), 1.69(3 \mathrm{H}, \mathrm{s})$ and $1.19(3 \mathrm{H}, \mathrm{t}, J=7.0) ;{ }^{13} \mathrm{C}-\mathrm{NMR}\left(\mathrm{CDCl}_{3}\right) \delta: 180.4,170.0,149.4,143.2,128.0,127.2,123.9,122.7,62.1$ 52.6, 41.0, 31.7, 18.8, 18.4 and 14.0 ; MS (EI) m/z(relative idensity \%) $262\left(\mathrm{M}^{+}, 82\right), 189(84), 171$ (100), 157 (73), 143 (71), 115 (36) and 91 (30); HRMS calcd. for $\mathrm{C}_{15} \mathrm{H}_{18} \mathrm{O}_{4} \mathrm{~N} \mathrm{~m} / \mathrm{z} 262.1205$, found m/z 262.1200. IR(KBr): $3380(\mathrm{br}), 2920,1730,1650,1200$ and $840 \mathrm{~cm}^{-1}$<smiles>CCOC(=O)C12C=CC(=O)C(O)=C1CC=C(CCC(C)=O)C2</smiles>

13a: Ethyl 8-hyd roxy-3-(4-methylpent-3-enyl)-7-oxo-1,4,4a,7-tetrahydronaphthalene-4acarboxylate

'H-NMR (CDCl3, $600 \mathrm{MHz})$ 8:6.96 (1H, d, $J=9.9), 6.53-6.45(2 \mathrm{H}, \mathrm{m}), 5.51-5.43(1 \mathrm{H}, \mathrm{m}), 5.11-5.05$ $(1 \mathrm{H}, \mathrm{m}), 4.13-4.05(2 \mathrm{H}, \mathrm{m}), 3.46(1 \mathrm{H}, \mathrm{d}, J=21.2), 3.05-2.95(2 \mathrm{H}, \mathrm{m}), 2.20-1.95(5 \mathrm{H}, \mathrm{m}), 1.72-1.53(6 \mathrm{H}$, $\mathrm{m})$ and 1.46-1.13 $(3 \mathrm{H}, \mathrm{m}) ;{ }^{13} \mathrm{C}-\mathrm{NMR}\left(\mathrm{CDCl}_{3}, 150 \mathrm{MHz}\right) 8: 180.3,169.9,149.5,143.8,134.6,131.9$, $127.2,127.1,123.8,118.6,62.2,52.3,38.2,37.2,26.0,26.0,25.6,17.7$ and $14.0 ; \mathrm{MS}$ (EI) m/z(relative intensity \%) $316\left(\mathrm{M}^{+}, 62\right), 247(43), 243(68), 175(88), 173(100), 157(23), 145(32), 115(24)$ and 69 (53); IR (NaCl): $3190,2920,2845,2380,1730,1645,1415,1375,1235,1205$ and $1180 \mathrm{~cm}^{-1}$ 
<smiles>CCOC(=O)C12C=CC(=O)C(O)=C1CC(CCC=C(C)C)=CC2</smiles>

13b: Ethyl 8-hydroxy-2-(4-methylpent-3-enyl)-7-oxo-1,4,4a,7-tetrahydronaphthalene-4acarboxylate

${ }^{1} \mathrm{H}-\mathrm{NMR}\left(\mathrm{CDCl}_{3}, 600 \mathrm{MHz}\right) \delta: 6.98(1 \mathrm{H}, \mathrm{d}, J=9.9), 6.53-6.45(2 \mathrm{H}, \mathrm{m}), 5.51-5.43(1 \mathrm{H}, \mathrm{m}), 5.11-5.05$ $(1 \mathrm{H}, \mathrm{m}), 4.27-4.13(2 \mathrm{H}, \mathrm{m}), 3.38(1 \mathrm{H}, \mathrm{d}, J=20.9), 3.11(1 \mathrm{H}, \mathrm{dd}, J=5.5,16.1), 3.05-2.95(1 \mathrm{H}, \mathrm{m}), 2.20$ $1.95(5 \mathrm{H}, \mathrm{m})$ and $1.72-1.53(6 \mathrm{H}, \mathrm{m}), 1.46-1.13(3 \mathrm{H}, \mathrm{m}) ;{ }^{13} \mathrm{C}-\mathrm{NMR}\left(\mathrm{CDCl}_{3}, 150 \mathrm{MHz}\right) \delta: 180.3,170.1$, $149.8,143.8,136.7,132.0,127.4,127.1,123.6,117.0,62.2,52.0,36.6,35.4,29.0,26.3,25.6,17.7$ and $14.0 ; \mathrm{MS}(\mathrm{EI}) \mathrm{m} / \mathrm{z}$ (relative intensity \%) $316\left(\mathrm{M}^{+}, 82\right), 243(40), 175(100), 173(87), 157(24), 145(20)$, 115 (22), 91 (13) and 69 (47); HRMS calcd. for $\mathrm{C}_{19} \mathrm{H}_{24} \mathrm{O}_{4} \quad \mathrm{~m} / \mathrm{z} 316.1675$ found $\mathrm{m} / \mathrm{z}$ 316.1665; IR $(\mathrm{NaCl}): 3382,2920,2845,2380,1730,1645,1415,1375,1235,1205$ and $1180 \mathrm{~cm}^{-1}$ 


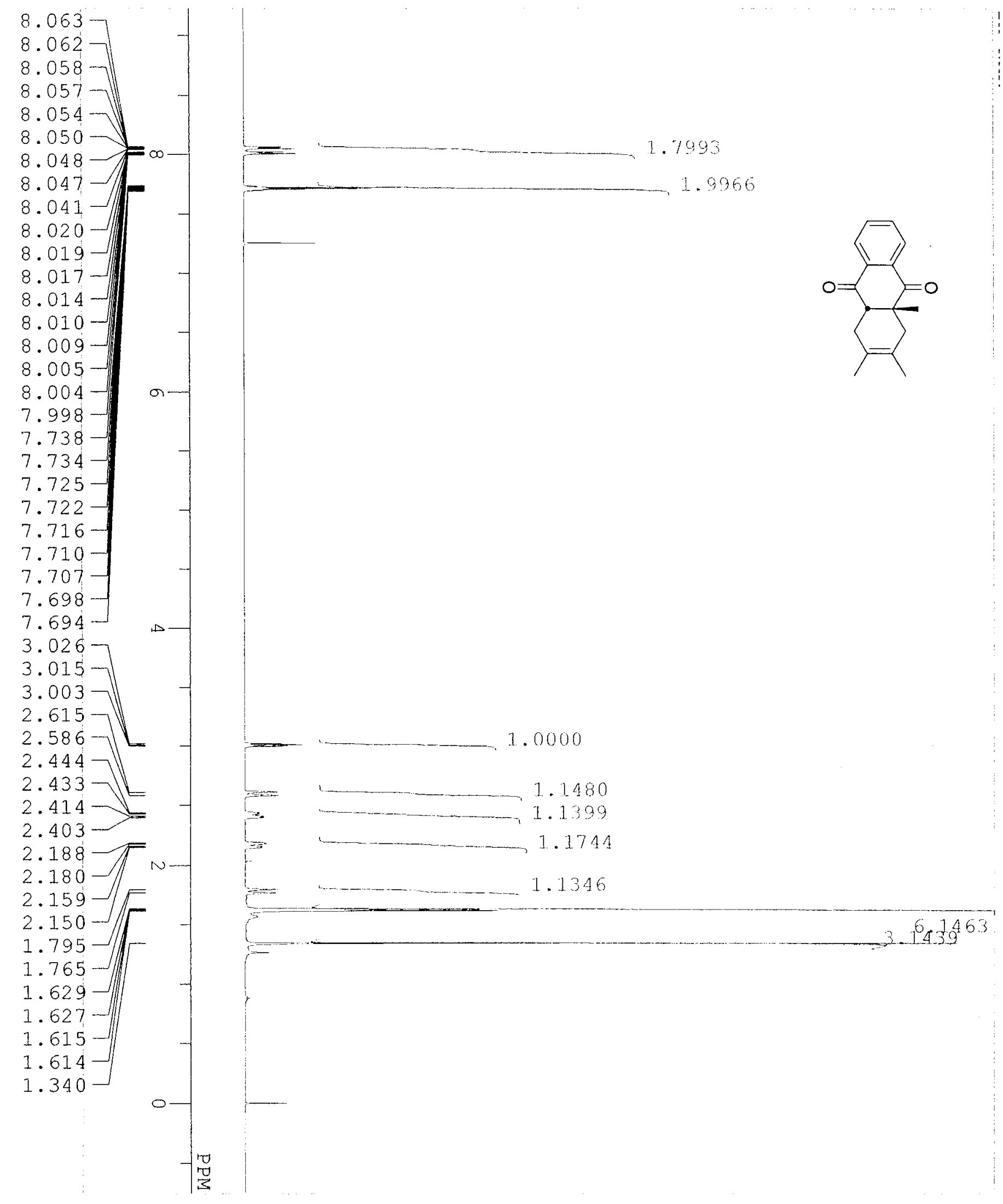




$$
1
$$




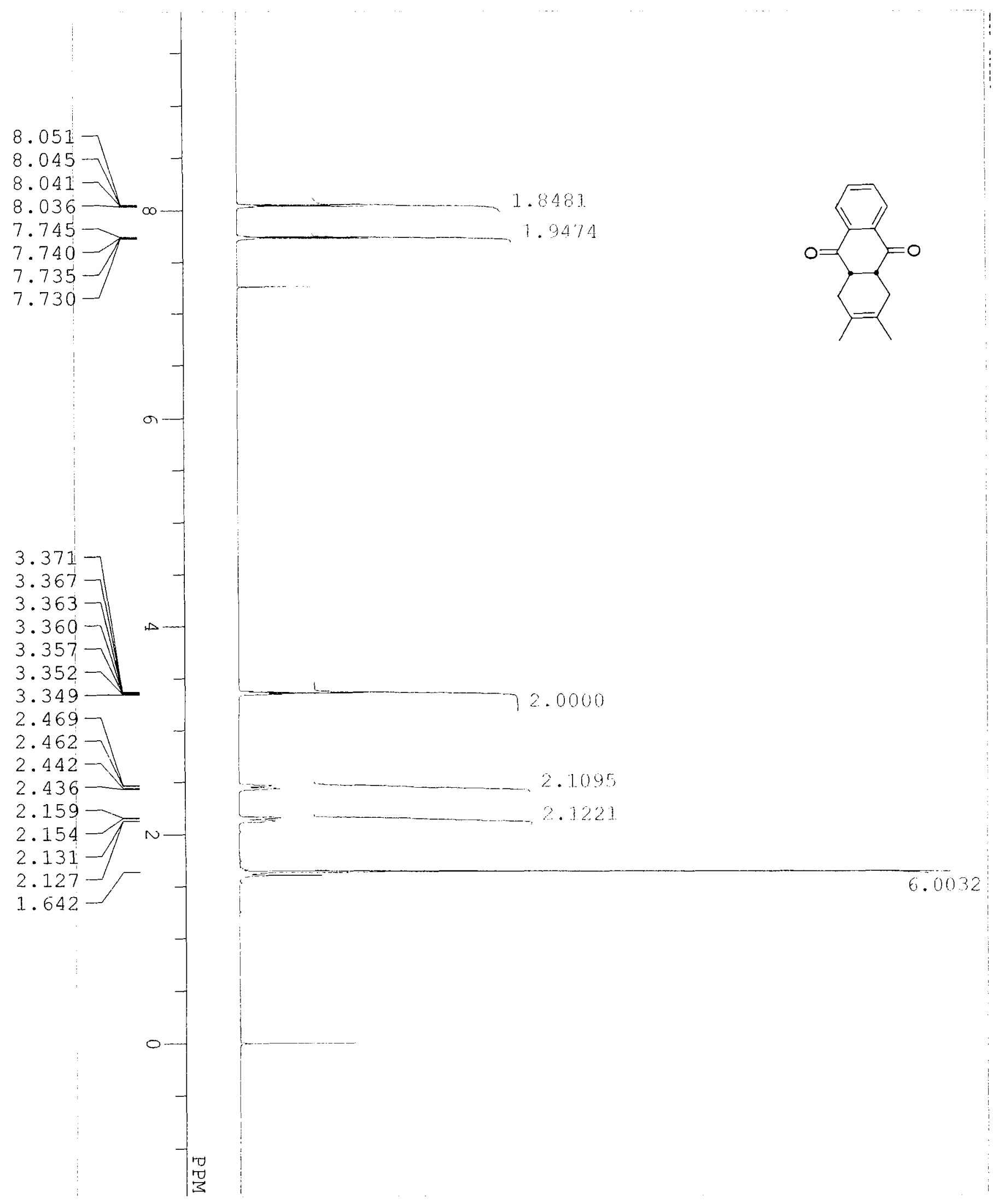




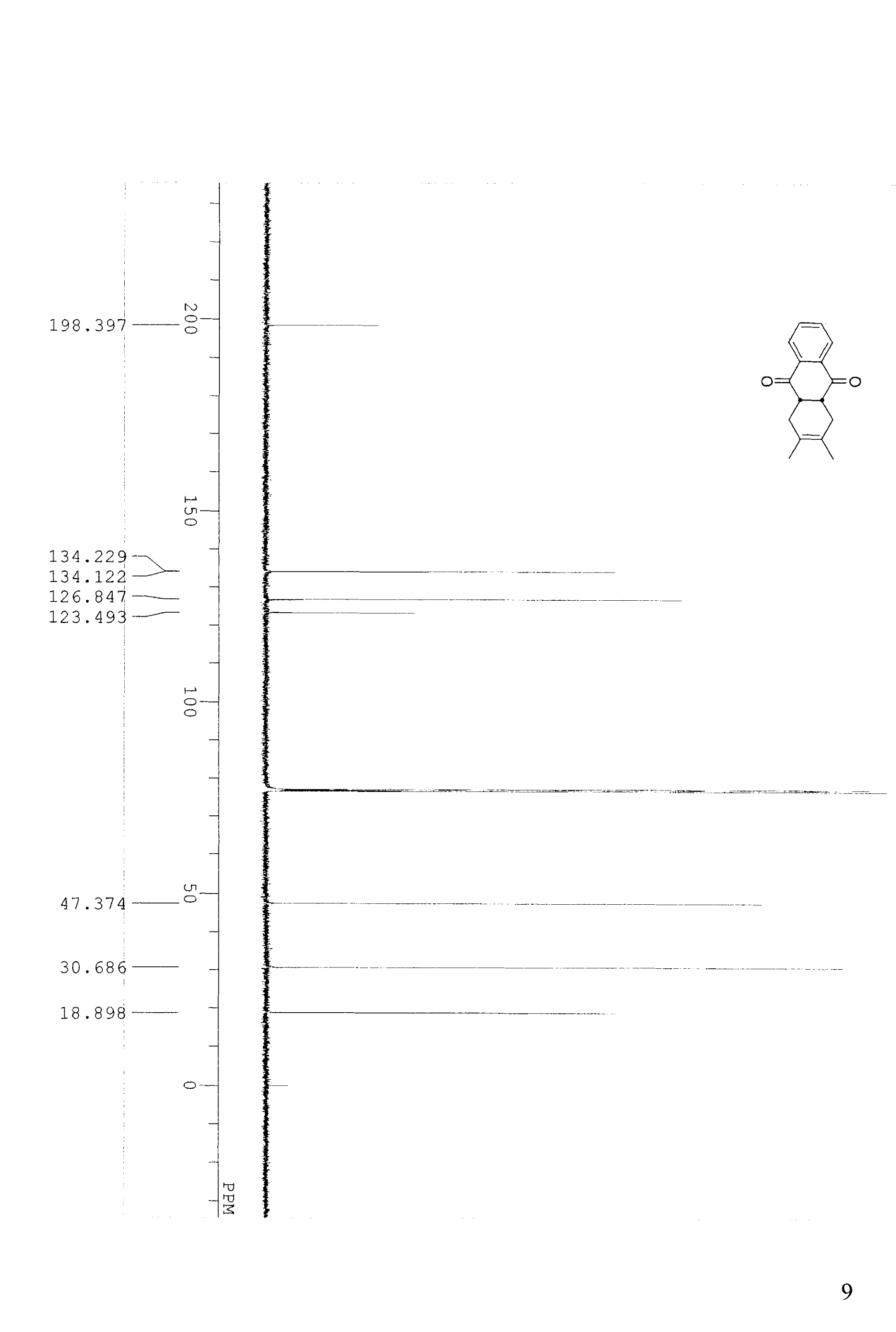




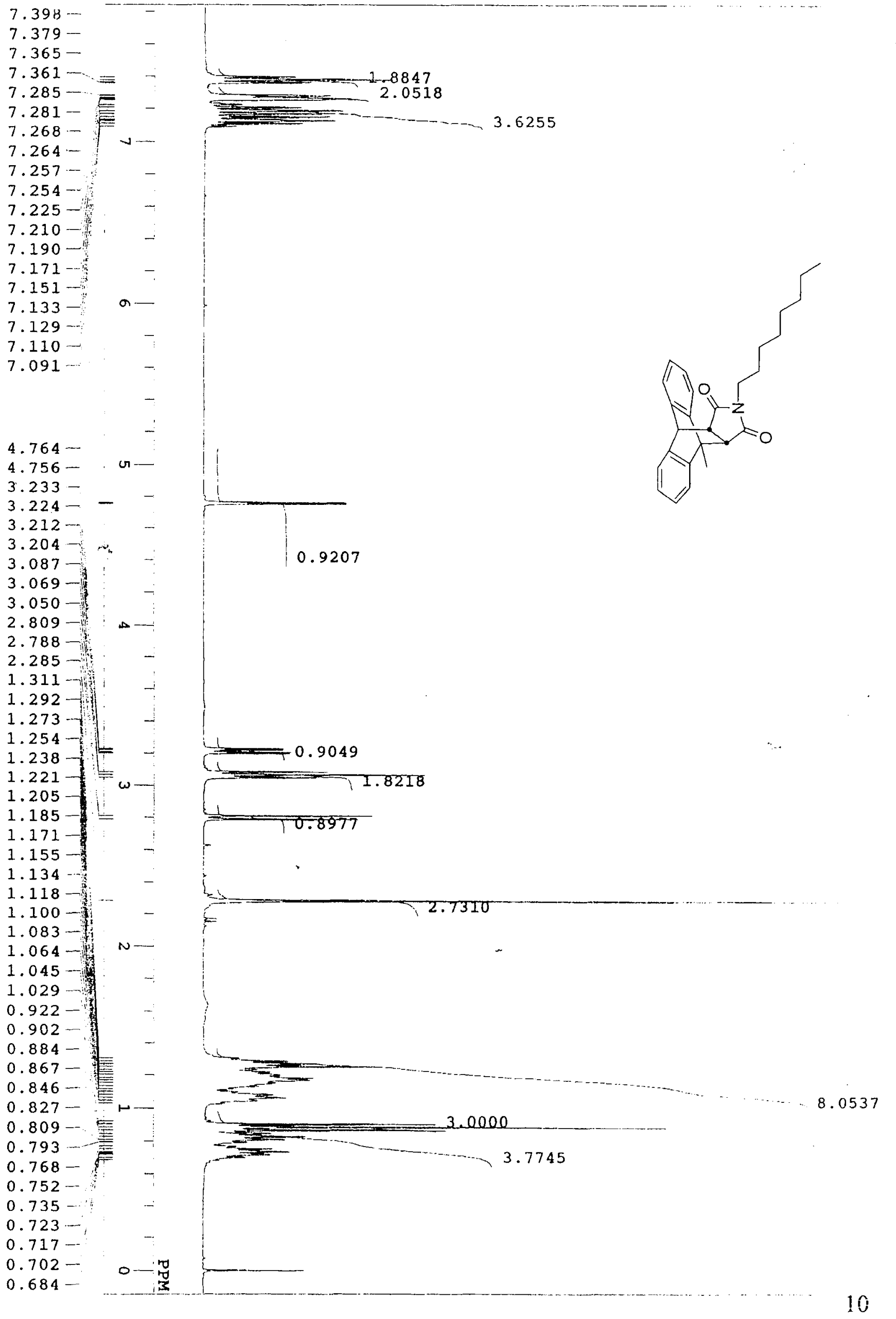




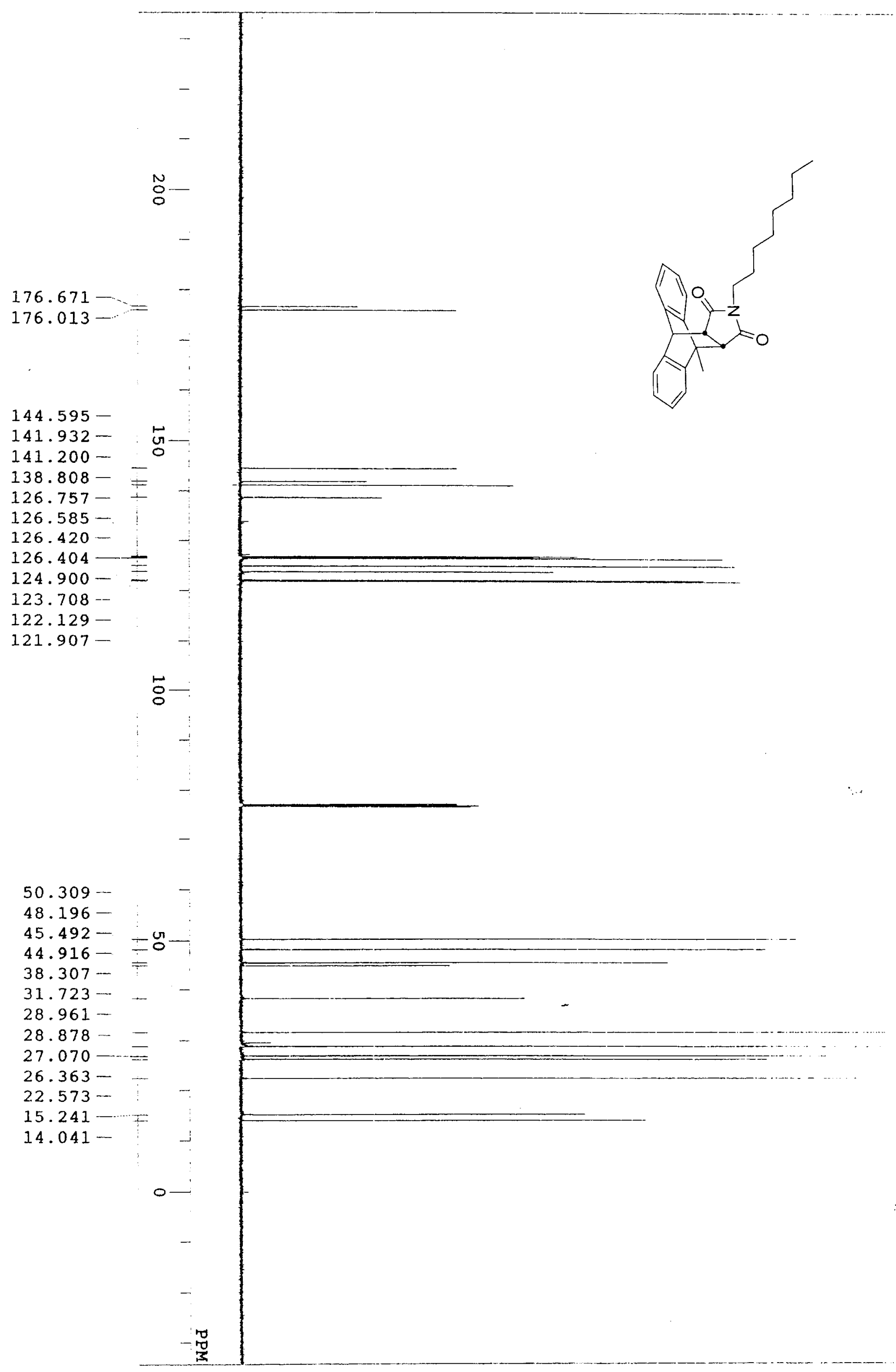




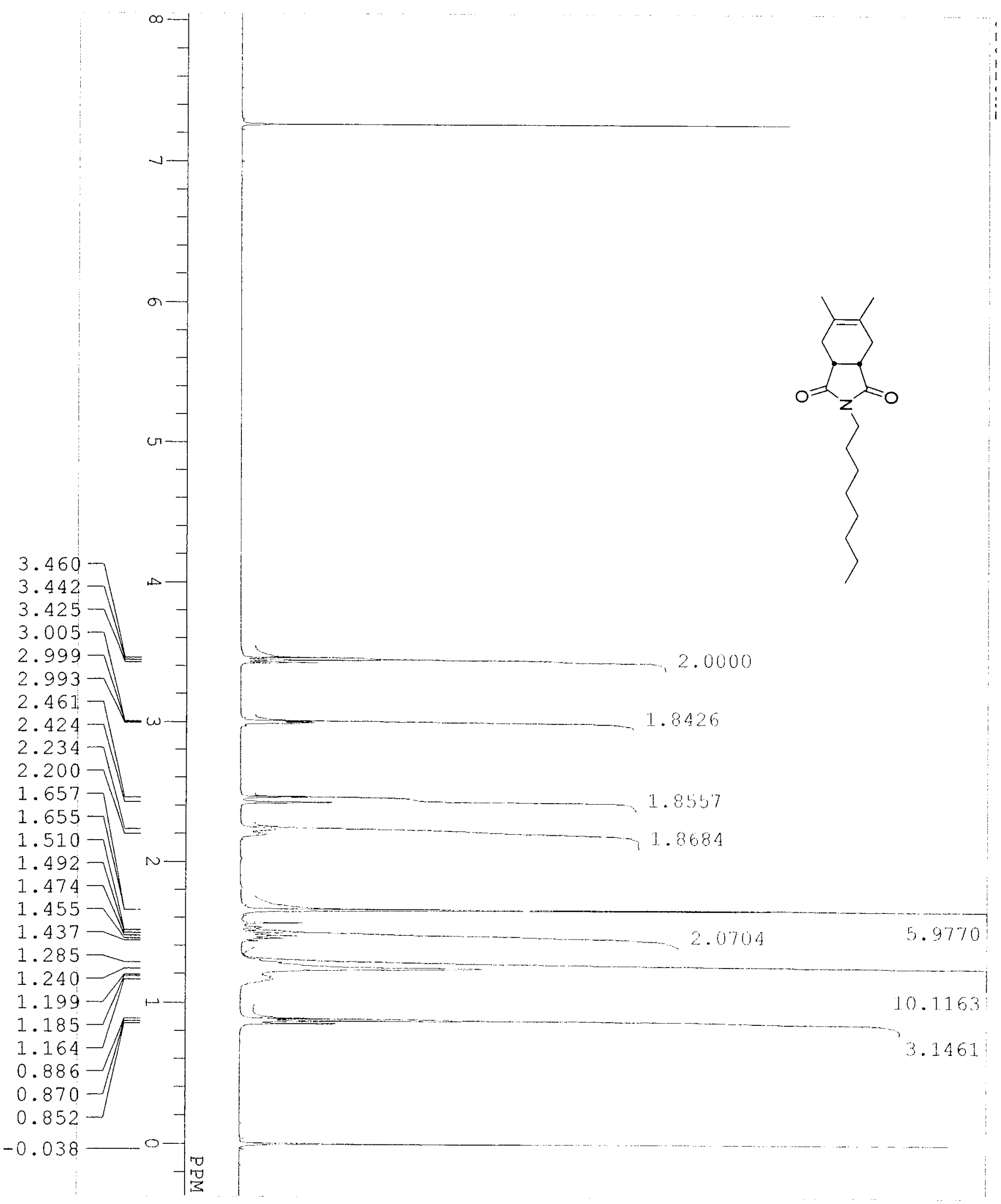




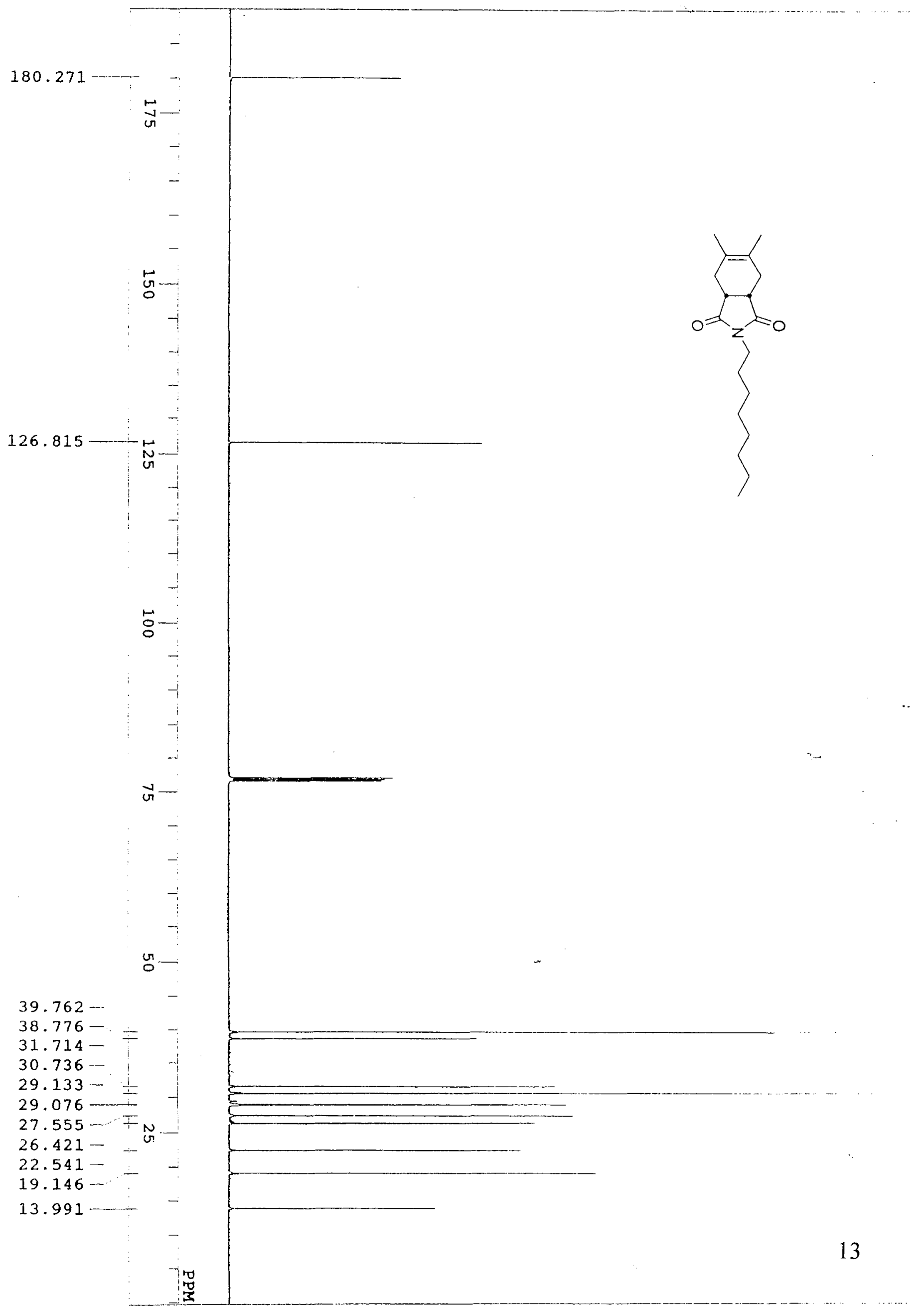




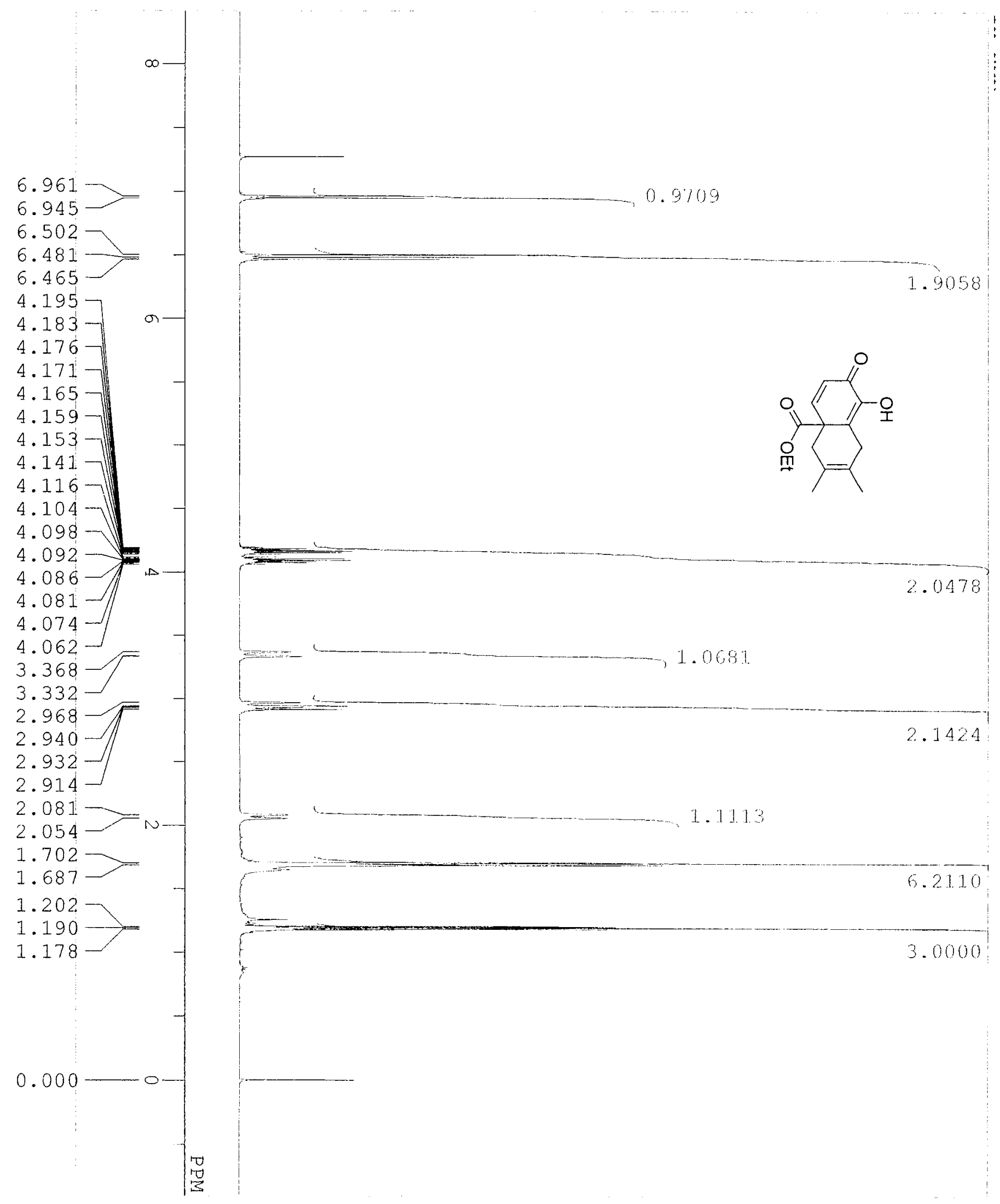




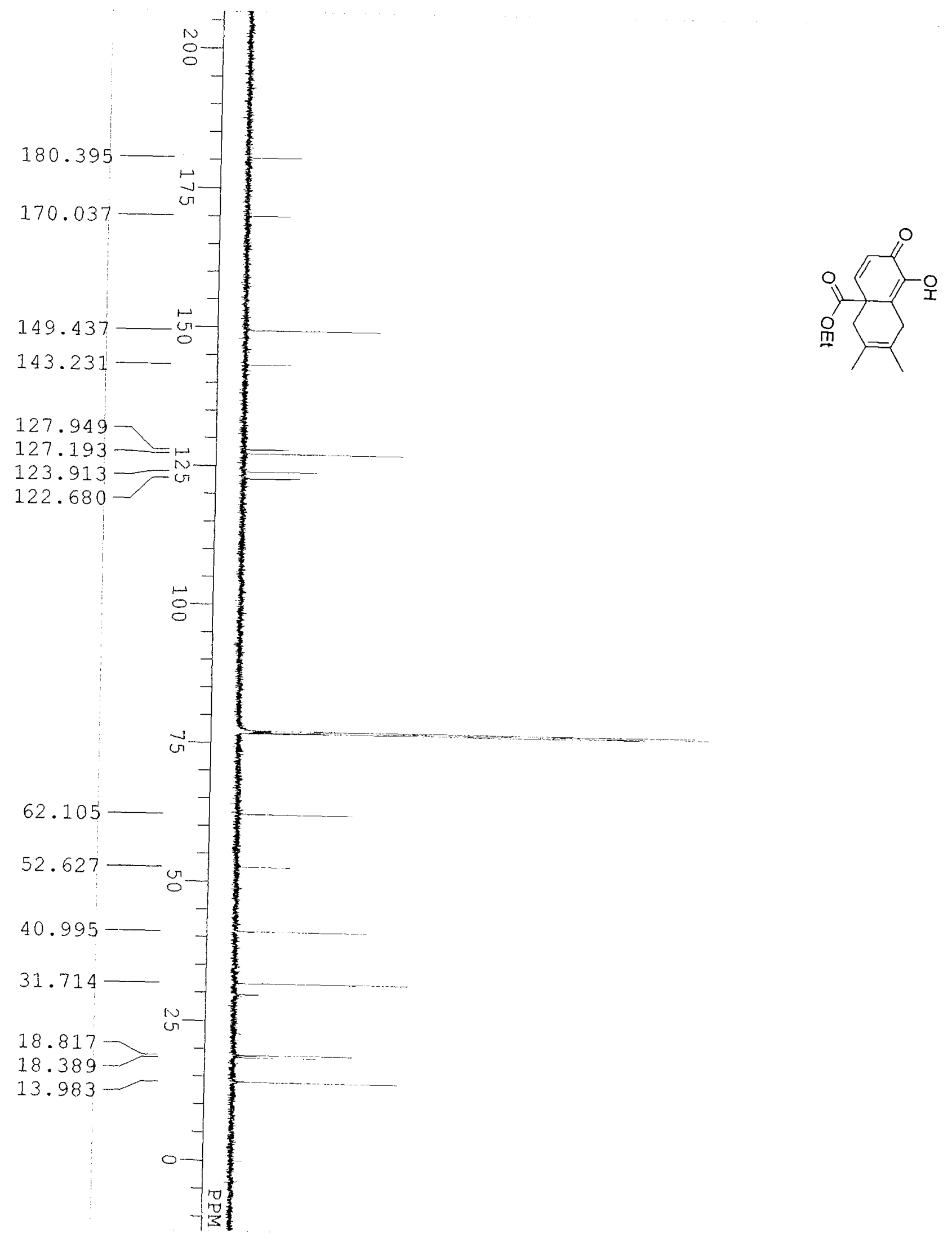




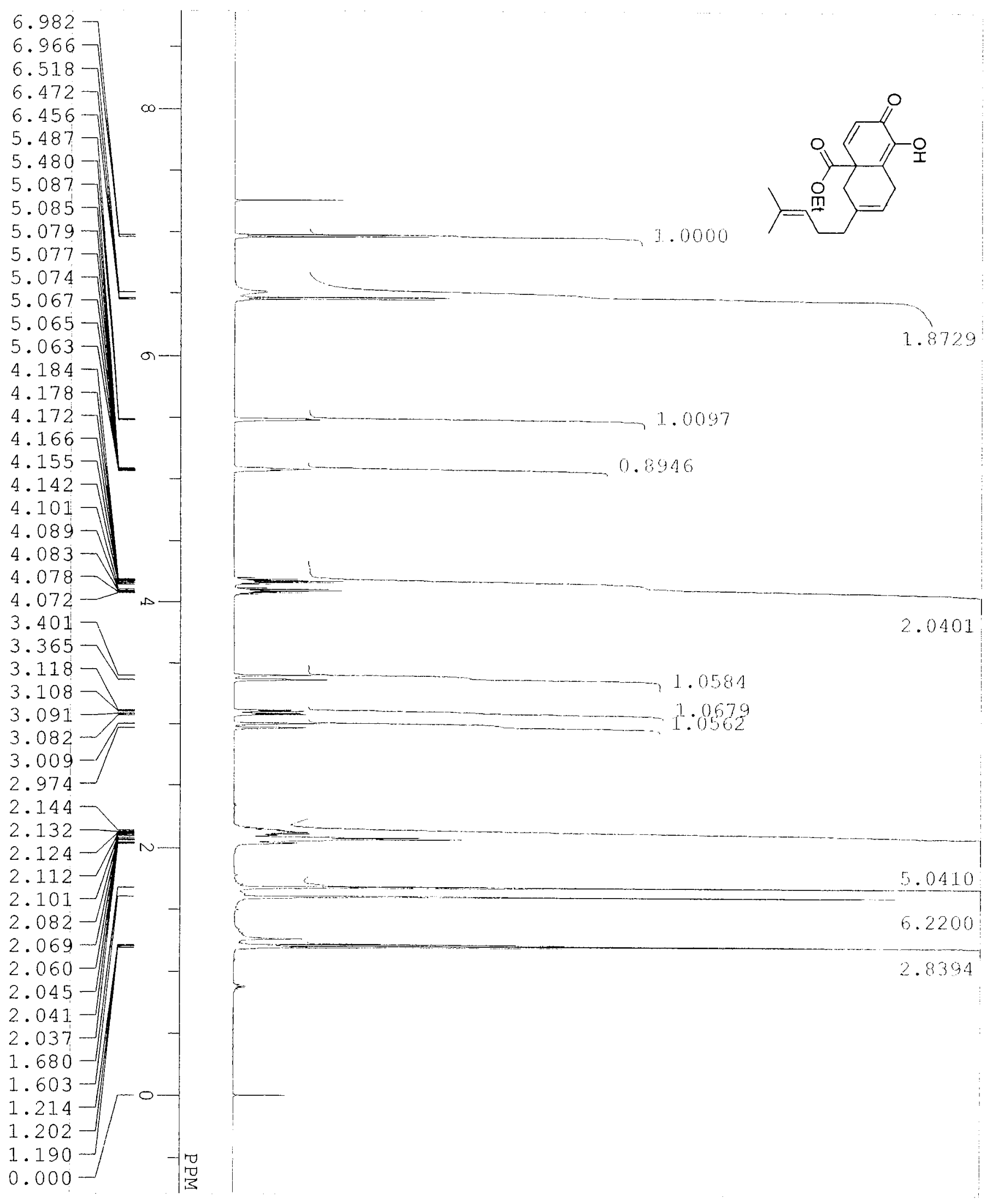




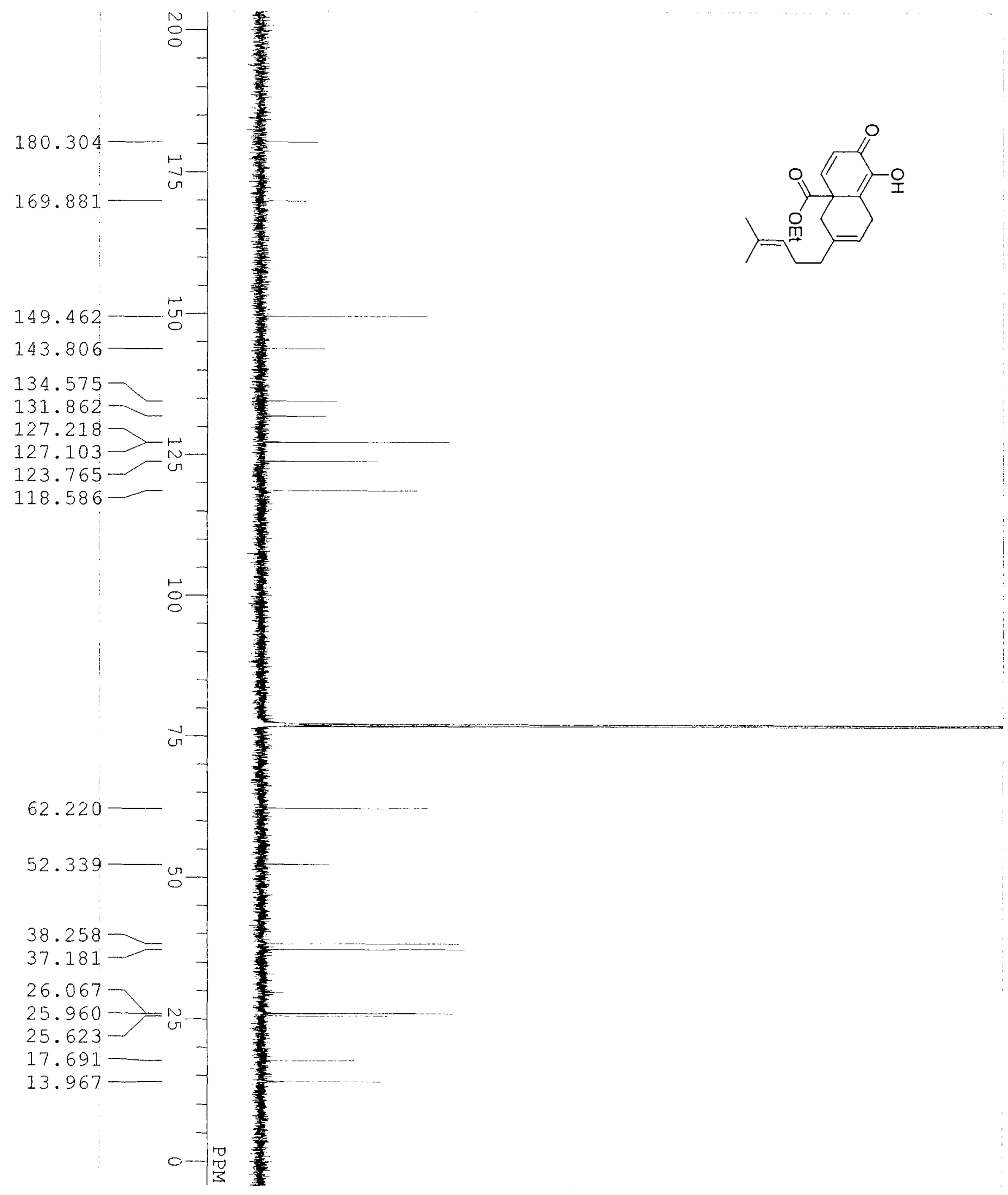




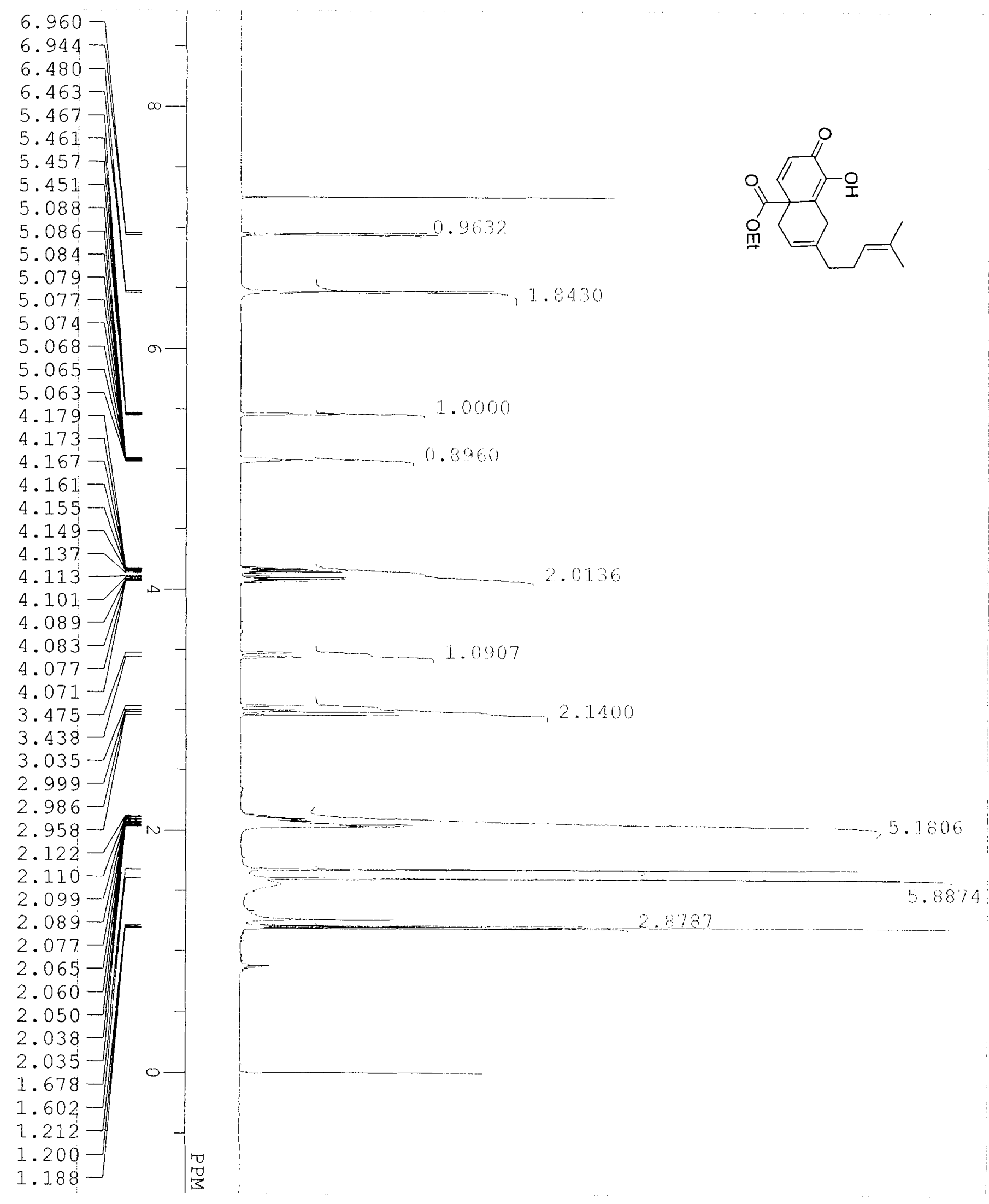




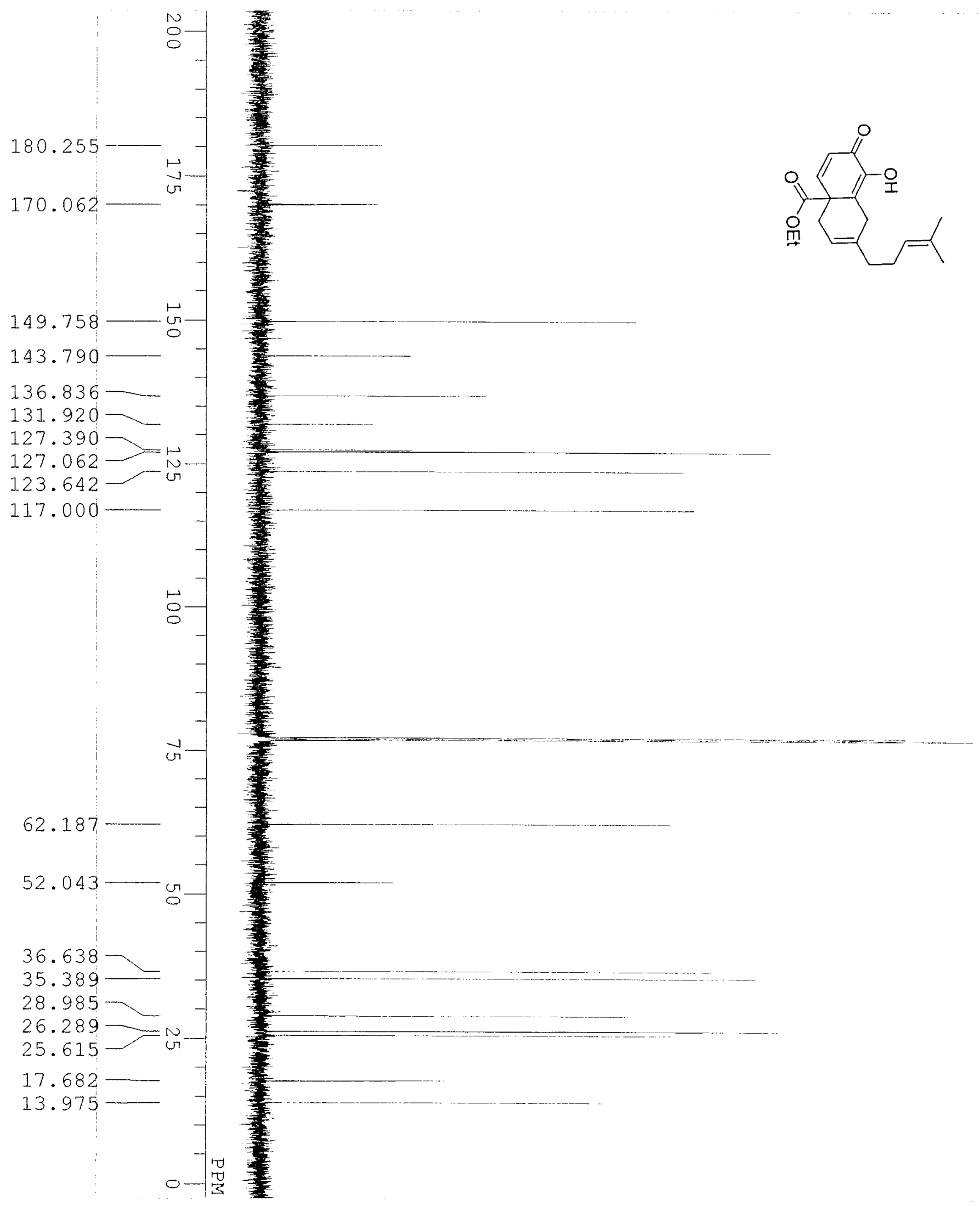

